\title{
Segregation and stability of a binary granular heap
}

\author{
A. G. Swartz · J. B. Kalmbach · J. Olson · R. J. Zieve
}

Received: 9 April 2008 / Published online: 9 April 2009

(C) The Author(s) 2009. This article is published with open access at Springerlink.com

\begin{abstract}
We measure stability of two-dimensional granular mixtures in a rotating drum and relate grain configurations to stability. We use two types of grains which differ in both size and shape, with the larger grains reaching a larger average angle before an avalanche. In our mixtures, the smaller grains cluster near the center of the drum, while the larger grains remain near the outer edge, a pattern suggesting that grain size rather than avalanche angle determines the segregation behavior. One consequence of the size segregation is that the smaller grains heavily influence the stability of the heap. We find that the maximum angle of stability is a non-linear function of composition, changing particularly rapidly when small grains are first added to a homogeneous pile of large grains. We conclude that the grain configuration within the central portion of the heap plays a prominent role in stability.
\end{abstract}

Keywords Rotating drum - Static sandpile - Avalanches . Segregation · Grain shape

\section{Introduction}

Granular materials have received much attention due to their important practical applications in fields ranging from geology to the food industry [1]. Despite the simple behavior of

This work was supported in part by the National Science Foundation's Research Experience for Undergraduates Program under PHY-0243904.

A. G. Swartz · J. B. Kalmbach · J. Olson · R. J. Zieve $(\varangle)$

Physics Department, University of California,

Davis, CA 95616, USA

e-mail: zieve@physics.ucdavis.edu individual grains, a granular system can display complex collective behavior. In different regimes of density and motion, granular matter may most mimic a solid, liquid, or gas [2]. Although a good deal of effort has gone into cataloging these phases, there is little understanding of how the microscopic grain configuration leads to specific macroscopic behavior; that is, which features in an arrangement of grains are most important. Observed hysteresis in the transition to crystalline order [3] and in the numbers of contacts between grains [4] demonstrates that the microscopic configuration contains subtle information on the system's history. A more striking illustration of the importance of microstructure comes from sound propagation experiments, where a fractional size change in a single grain can change the total sound transmission through a granular heap by up to $25 \%$ [5].

Understanding the connection between configuration and behavior is particularly necessary for moving from experimental studies of homogeneous mixtures to the heterogeneous mixtures that appear in most industrial and other applications, since the range of possible configurations is broader for a mixture than for a homogeneous sample. In addition to slight shiftings of grains between stable configurations, the distribution of the two species through the sample can vary. In fact, heterogeneous mixtures commonly undergo segregation, a phenomenon of great interest for materials processing [6]. Segregation of binary mixtures also occurs in the laboratory, in vibrating bed $[7,8]$ and rotating drum experiments [9-22], and upon pouring into a Hele-Shaw cell [23-25]. It can be caused by differences in particle size, shape, smoothness, or density. Here we measure binary mixtures in a two-dimensional rotating drum. We describe our apparatus and the segregation patterns we observe. We then combine the segregation observations with measurements of avalanches to probe how different configurations influence stability. 


\section{Experimental setup}

The grains of our mixture are composed of $\frac{1}{8}^{\prime \prime}$-diameter ball bearings. We use both single spherical bearings and hexagonal shapes created by welding seven ball bearings together, as described elsewhere [26]. The two grains are shown in the inset of Fig. 2. The hexagonal shapes can form near-perfect triangular lattices of the individual spheres, in which the joints between balls are impossible to distinguish, demonstrating that the welding process does not significantly distort the balls.

We use a rotating drum consisting of a $\frac{1}{8}^{\prime \prime}$-thick aluminum sheet with a $13.96^{\prime \prime}$-diameter circle cut out of it. Two $\frac{1}{2}{ }^{\prime \prime}$-thick sheets of Plexiglas sandwich the aluminum sheet and contain the mixture. We believe that friction between the grains and the Plexiglas is not a major factor in the behavior of the pile. Our measured angle of stability for a pile of spherical grains is $31^{\circ}$, very close to the $30^{\circ}$ expected from simple geometrical arguments for a two-dimensional heap of circles [27]. Also, since we use brass or steel balls, gravitational forces in our system are stronger than in piles of glass or plastic beads. Confining the grains to a single layer allows us to track visually the exact configuration of the grains during the rotation. It also eliminates the axial segregation into bands that has been observed in three-dimensional experiments and models of binary mixtures $[10,13,17,19,20,22]$. We can then focus purely on radial segregation, which also occurs in three-dimensional rotating drums $[12,15]$ in a wider range of conditions than axial segregation.

The drum rotates about its center with rotational speed controlled by a motor. We rotate at $500 \mu \mathrm{Hz}$, or one full turn in just over half an hour. This slow speed allows for staticlimit behavior, characterized by discrete avalanches and a near-linear free surface. An avalanche occurs when the pile rotates into an unstable configuration, at which point grains begin to flow down the pile. We refer to the angle of the pile's free surface when an avalanche begins as the angle of stability, and the angle after the avalanche as the repose angle. If one configuration leads to a larger angle of stability than another, we refer to it as a "more stable" configuration. During an avalanche only the top few layers of grains flow and the bulk of the heap remains stable. The flowing layer takes the shape of a pie wedge determined by the stability and repose angles: more grains flow at the top and less at the bottom. In a typical avalanche for our piles, which fill about $28 \%$ of the container, the flowing layer reaches a maximum depth of about $1-2 \mathrm{~cm}$. The rotation of the drum during an avalanche is about one-third of a degree, for most purposes small enough to be ignored. We also verified experimentally that, within the uncertainty of our measurements, the distribution of avalanches in angle does not change with rotation speed up to $1 \mathrm{mHz}$.
The binary mixture is inserted into the tumbler through a slot in one side of the aluminum sheet. The opening is closed during rotation. Since we are studying the behavior of binary mixtures, the hexagonal shapes must be entered carefully to avoid breakage. As described below, even a small percentage of single spheres in a pile of hexagonal grains noticeably decreases the pile's stability, and broken hexagons have a similar effect. After completing measurements on a pile, we check for broken shapes. If more than $1 \%$ of the hexagonal shapes are broken, we discard the data and remeasure that composition. The breakage leads to a slight uncertainty in the effective mixture composition. Since the mixtures we measure have nominal compositions from 0 to $100 \%$ hexagons, and differ from each other by at least $2 \%$, an uncertainty in composition of less than $1 \%$ does not qualitatively affect our results.

An alternate method for loading the mixture is to disassemble the tumbler and remove one of the Plexiglas faces. The shapes can then be placed in the aluminum sheet with little risk of breaking. However, if the screws which hold the Plexiglas and the aluminum sheets together are not tightened equally, slight differences in how uniformly the grains are confined to a single layer cause changes in avalanche angle with container orientation. The signature of this problem is a periodicity in the average angle of stability that matches the rotation rate of the drum. To avoid such bias, we adjust the container until our data show no rotation periodicity, and then leave the drum undisturbed through the entire set of measurements.

To achieve "steady-state" measurements, where each grain configuration has developed from previous avalanches, we rotate at $500 \mu \mathrm{Hz}$ for at least $20 \mathrm{~min}$ after a mixture is loaded into the tumbler before beginning to record avalanches. This avoids anomalous results arising from the configuration of the grains upon loading. We then rotate the drum for one to two hours to obtain about 100 discrete avalanches, filming the entire time with a digital video camera. Avalanches are larger and less frequent for the hexagons than for the spheres, so the filming takes correspondingly longer. We transfer the frames immediately before and after each avalanche to a computer. For each image we perform a series of vertical scans that identify the height of the free surface of the pile. Connecting the heights corresponding to the different scans gives a piecewise linear curve that tracks the free surface. We then extract the average slope by a least-squares fit to the heights. In this manner we find both the angle of stability before an avalanche and the angle of repose afterward. For further analysis, we use a minimum 4-degree difference between the two angles as a cutoff in avalanche size, discarding any images from smaller rearrangements. As described elsewhere [28], we can also compute the pile's packing fraction from the images. White paper around the outside of the circular drum region and a red 

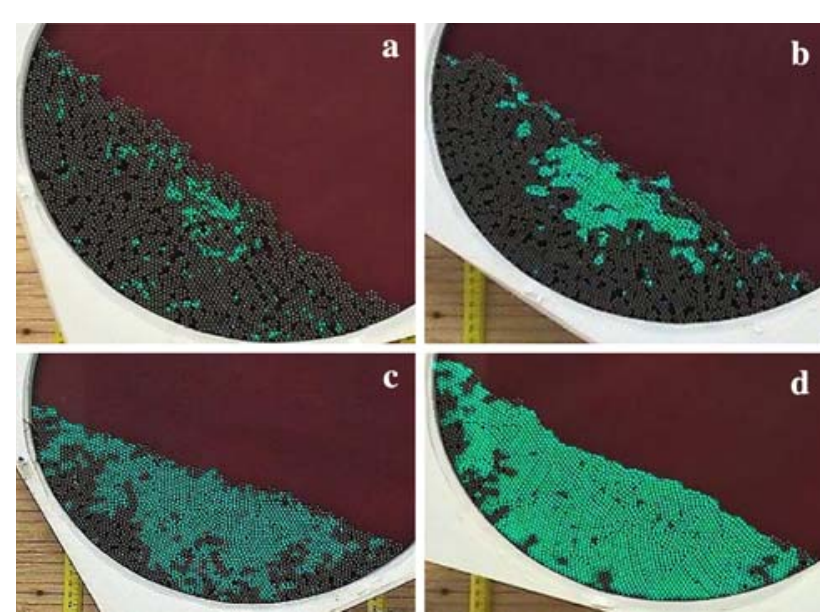

Fig. 1 Typical segregation for mixture of hexagons (dark) and spheres (light) in steady-state rotation. Shown are mixtures with a 7\%, b $18 \%$, c $58 \%$, and $\mathbf{d} 89 \%$ single spheres by area

background inside provide contrast that improves the image processing.

For many of the measurements we use brass ball bearings for the single balls, which we can distinguish by color from the steel hexagons. In Fig. 1, we show typical configurations of the mixtures. To better convey the configurations in these small images, the brass balls are lightened (green online) for visibility [29]. We identify the brass balls from their yellowish color. About $70 \%$ of the brass balls are determined unambiguously. However, light reflected from nearby brass balls or red background can give even the steel balls a slight yellow tint, producing a small overlap in the color ranges of the brass and steel balls. We select those balls with the strongest yellow tint as brass, choosing a cutoff so that the correct number of brass balls are found. For a few piles we have compared the computer determination with the original pile, and we find that about $95 \%$ of the brass balls are properly identified. The few misidentifications do not qualitatively alter the segregation patterns.

\section{Results and discussion}

\subsection{Segregation}

The spheres cluster near the center of the drum, usually slightly below the free surface of the heap. The hexagons tend to inhabit the outer portions of the drum. Several previous studies $[16,19,22]$, both numerical and experimental, have also found that size differences lead to radial segregation, with smaller shapes moving to the drum center. Interestingly, other work $[14,18]$ shows that differences in stability angle can also cause radial segregation, with shapes of higher stability moving to the center. The size and shape characteristics can compete with each other. When a mix of small smooth grains and large faceted grains are dropped onto a pile, the grain types form striations within the pile [23-25]. A much simpler segregation occurs when the smaller grains are also more faceted. In this case the larger shape collects at the bottom and the smaller shape at the top of the pile, with a sharp demarcation between the two regions [24]. The upper portion of the pile has a steeper slope, as expected from larger angle of stability of the more faceted grains. Geologists have long been aware of such patterns, using striations to understand how sediment settled out of the flows that created rock structures [30].

For our shapes, the hexagons with their irregular edges have a higher angle of stability than do homogeneous spheres. In the experiments mentioned above on building a pile from a grain mixture, the segregation pattern depends on the difference in stability angles. This also occurs for continuously avalanching material in a rotating drum, where a characteristic " $\mathrm{S}$ "-shaped surface profile $[31,32]$ naturally causes the component with higher repose angle to collect in the central, steeper portion of the pile. For components differing in shape, the less regular material generally has a higher repose angle and moves to the center. However, for our hexagons and spheres, the size difference apparently overwhelms shape effects in the segregation process. We find no striations or fingering, but rather a clustering consistent with pure size segregation. Possibly this behavior would change for large rotation speeds, but at our slow speeds, with a near-linear free surface, the reduced influence of shape is not surprising.

Since the brass balls we use weigh about $10 \%$ more than the steel balls, the change in material could affect the segregation and avalanche behavior. For a few mixtures we repeated our measurements with all steel balls, and verified that substituting the brass ball bearings does not change either the avalanche angles or the packing fractions. We have also recently observed similar segregation patterns with all steel balls, where the hexagons were painted with green nail polish to identify them. Hence the density difference of the materials appears to be a relatively unimportant factor in our results.

Figure 1 shows the progression of the segregation as the concentration of single balls increases. For small percentages (2-8\%) the singles, which converge towards the bottom of the laminar layer, group themselves into small clusters. The size and number of these clusters increases with composition. The 7\% composition (Fig. 1a) shows singles congregating into these distinct clumps of 5-10 singles. These clusters all lie within an elongated region running roughly parallel to the free surface.

At $18 \%$ spheres (Fig. 1b), the small clusters have grown together and the singles have saturated the elongated area near the center of the free surface. With further increase in the fraction of single spheres, this central region grows, spreading outward both along the free edge and perpendicular to 


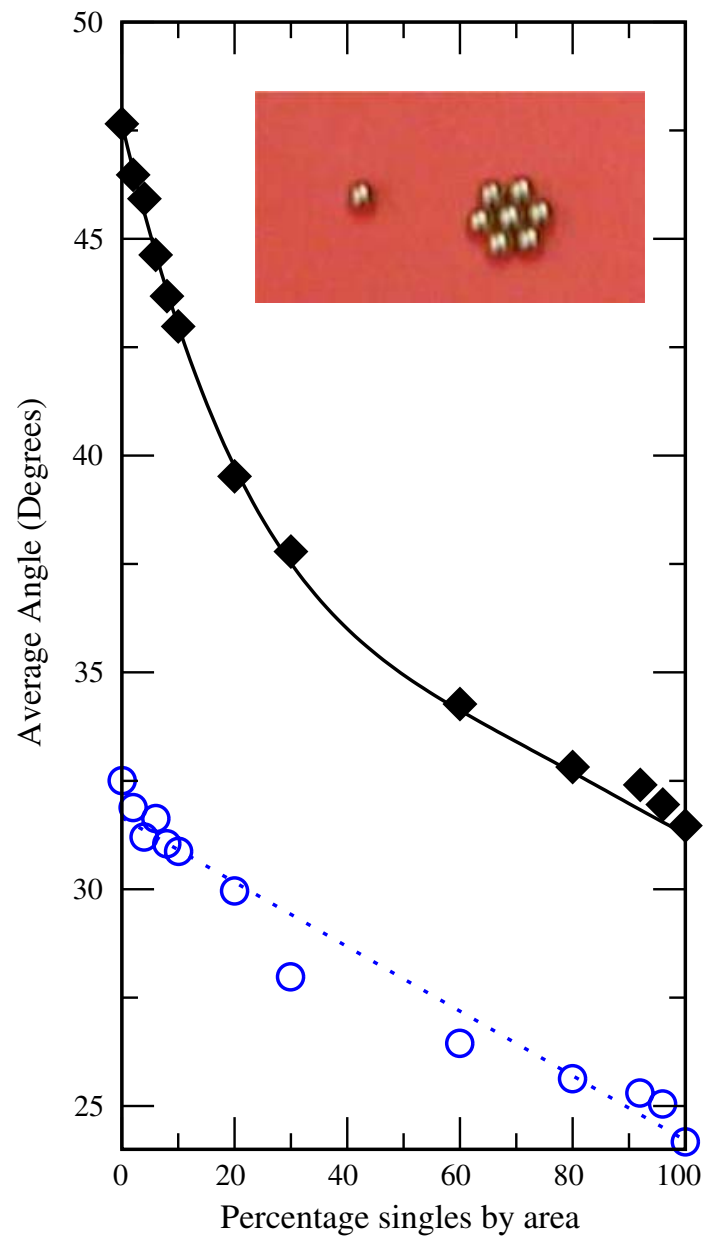

Fig. 2 Angles of stability (filled diamonds) and repose (open circles) for different pile compositions, averaged over about 100 avalanches for each pile. One standard deviation for the average angle is typically less than half a degree, so error bars would be comparable to the symbol size and are not shown. The solid line is a guide the eye. The dotted line, which is the best linear fit to the repose angles, is shown to illustrate the downward dip for intermediate compositions. Inset: sphere and hexagon grains

it. At the other extreme, in mixtures with primarily single spheres (Fig. 1d), the few hexagons lodge along the outer edge of the container.

\subsection{Stability}

We now turn to the maximum angles of stability for our binary mixtures. From previous work [28] we know that hexagons are significantly more stable than single ball bearings. Similar results were reported in [33] for pentagons. The maximum angle that a pile attains before an avalanche varies depending on the exact arrangement of grains within the pile, with the standard deviation typically four to five degrees. In Fig. 2, we average the angles before approximately 100 avalanches for each composition. The standard deviations of the average values are comparable to the symbol size. We find that varying the fraction of spheres in the mixture changes the stability of the pile in a decidedly nonlinear manner. For mixtures with a small percentage of spheres, the stability angle decreases quickly. At the other extreme, a few hexagons in a pile of spheres have much less effect on the pile's stability. The repose angles are also shown in Fig. 2, with a dotted line representing the best linear fit. The dependence of repose angles on composition is much closer to linear, although here too the angles dip downward for the most evenly mixed compositions.

The addition of spheres to the pile of hexagons serves to decrease the angle of stability, which is unsurprising given the much lower angle of stability of homogeneous spheres. The segregation behavior described above may be one possible factor in explaining why small numbers of single spheres have a larger effect on the observed angles of stability for a hexagonal pile than a few hexagons do on a pile of spheres. We propose that when avalanches begin depends most heavily on the configuration in the central region of the drum. Since the spheres tend to cluster in the middle of the pile, a small concentration of single spheres could have an enhanced effect on the avalanches. Conversely, a small number of hexagons in a pile of spheres move to the outside of the drum and would have much less effect on the avalanche behavior.

\subsection{Effects of segregation on stability}

To test the importance of the central portion of the pile, we examine the stability of configurations far from the typical segregation patterns. Using a mixture of $91 \%$ hexagons and 9\% spheres, we load the spheres into the drum first, so that they cluster along the bottom edge of the drum, and then add the hexagons on top of them. This places the spheres as far as possible from their eventual position in the center just below the free surface. We record avalanches from when rotation begins, without the usual 20 -min settling time. Since the single balls are peripheral for the first few avalanches, we expect stability comparable to the initial stability of a homogeneous hexagonal pile. During these early avalanches, the singles rotate with the drum until they reach the top of the pile and begin participating in the avalanches. Next we expect a transition period in which the spheres are no longer extraneous and the stability angle should decrease. Once the single spheres have migrated to their usual central position, subsequent avalanches should reflect the usual characteristics of the steady state of the mixture.

Figure 3 shows the first 15 avalanches in this test of initial stability for the mixture with $9 \%$ spheres, as well as a similar measurement of initial avalanches for a homogeneous pile of hexagons. Because of the variability in individual avalanches, we measure the stability angles in several trials at each composition. Between trials the drum is emptied and 


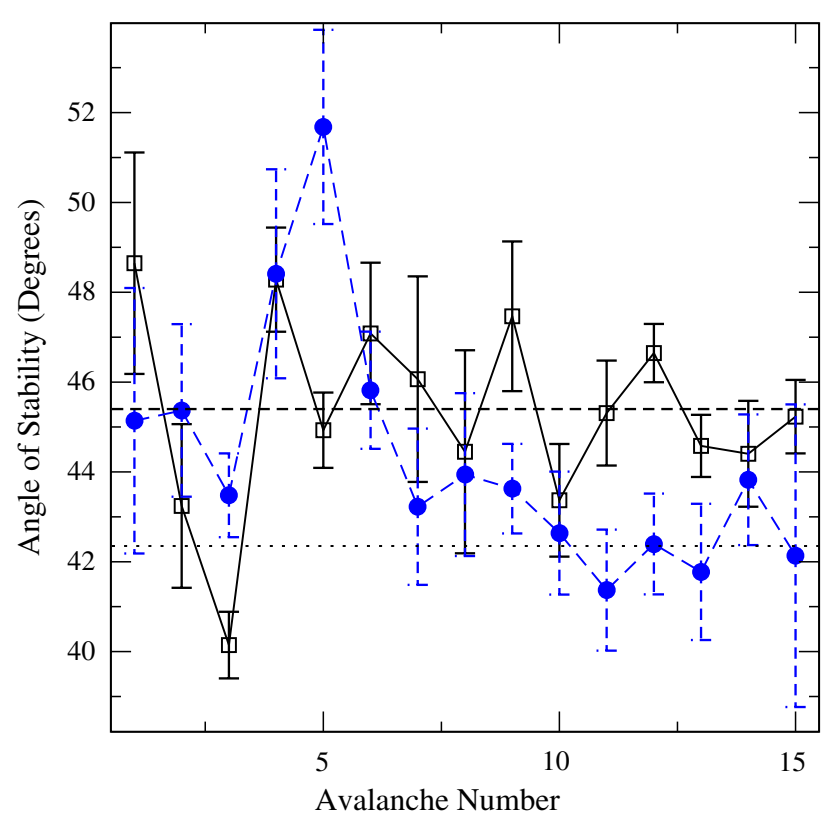

Fig. 3 Avalanche stability angle for the first 15 avalanches for homogeneous hexagons (open squares, solid line) and a mixture with $9 \%$ singles (filled circles, dashed line). Error bars correspond to one standard deviation in each direction. Horizontal dashed and dotted lines are averages of the last six avalanches for the pure hexagon and the mixture, respectively. Data are averaged over five runs for the hexagons and six for the mixture. The mixture was loaded for each run with the single spheres along the outside edge of the container, as described further in the text

reloaded. The data of Fig. 3 are averaged across these trials, avalanche by avalanche. Standard deviations are large compared to those of Fig. 2 because of the fairly small number of trials: six for the mixture and five for the pure hexagons.

Even for the homogeneous pile, the first few avalanches depend on the packing produced by loading the drum rather than on configurations resulting from previous avalanches, and show wide variations in the maximum angle before the avalanche. The similar behavior for the first few avalanches in the homogeneous pile and in the mixture shows that the large variations stem not from the particular location of the single balls around the container edge but from more general effects of loading the drum. The first six avalanches of both compositions have average angle of stability comparable to that of the steady-state homogeneous hexagons. This indicates that, initially, the singles are indeed peripheral both physically and in their effect on the avalanches. After about 10 avalanches, the average stability for the $9 \%$ composition has transitioned towards the steady-state average. Visually, this corresponds well to when the single spheres permeate the central area of the mix and start the clumping characteristic of radial segregation. The segregation occurs within about $\frac{1}{3}$ of a full rotation.

The initial stability measurements confirm the importance of the central region of the drum avalanches, but deliberately

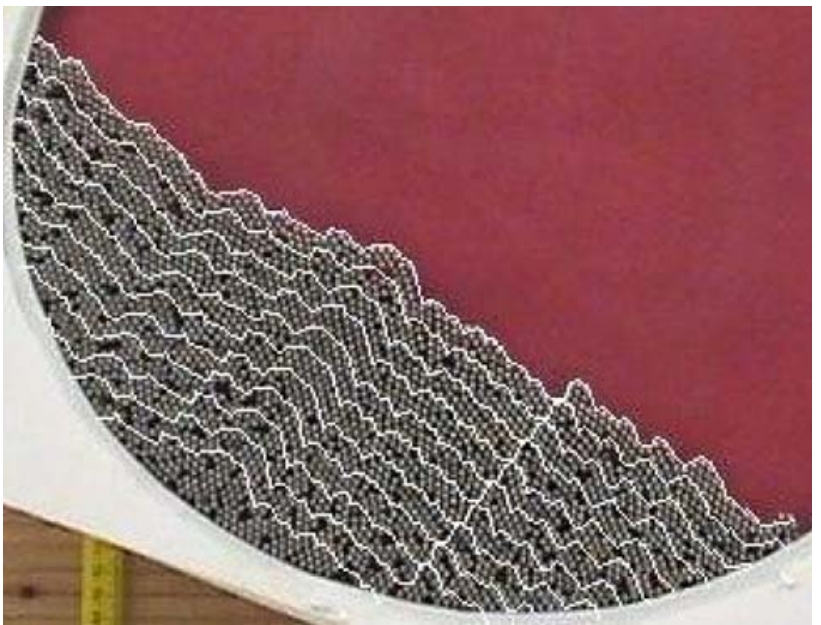

Fig. 4 An illustration of how the pile is divided into slices, following the description in the text

arranging the grains to test the stability of certain arrangements is a painstaking process. We can also study naturally occurring variations in grain configurations and how they correlate to stability. In this case we cannot choose specific arrangements to examine, and the distinctions among piles are less extreme; for example, the single spheres always clump near the center. On the other hand, generating large numbers of avalanches requires far less labor.

To investigate the importance of individual single spheres, we divide each pile into slices as illustrated in Fig. 4. In defining the angle of the free surface, we already identify a piecewise linear curve that tracks the irregularly shaped edge of the pile, shown as the uppermost white curve in Fig. 4. We then translate the irregular curve perpendicular to the surface in 1-cm increments, with the translates also shown in white, and count the number of brass balls between each pair of adjacent curves. The inset to Fig. 5 shows what fraction of the single balls reside in each slice, averaged over all avalanches for a given mixture. Combined with the total number of singles in the pile, this gives an absolute measure of the number of single near the free surface. Among the three concentrations shown, the segregation phenomenon is strongest for the pile with $18 \%$ singles.

For each pile composition and slice depth, we calculate the correlation between the number of brass balls found in the slice and the angle of the avalanche for that configuration. The main frame of Fig. 5 displays the results. The trend, clearly seen for the pile with $18 \%$ singles, is that for the slices nearest to the free surface, the number of single balls is negatively correlated with the average angle or stability. When many single balls are close to the surface, a pile is more prone to avalanche. For slices deeper in the pile, the correlation coefficient changes sign, which is natural: since a pile has a fixed number of single balls, if the number well 


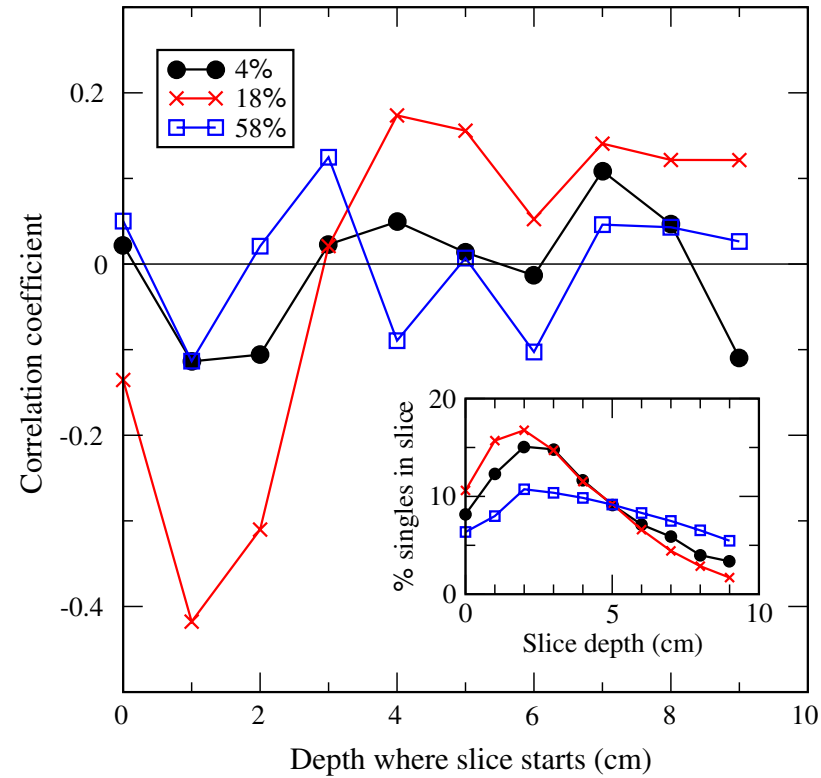

Fig. 5 Correlation between avalanche angle and the number of single balls in a slice of the pile. Slices are $1 \mathrm{~cm}$ thick, and the values on the horizontal axis indicate the distance from the upper edge of the slice to the free surface of the pile. Correlation coefficients are normalized to \pm 1 for perfect correlation. Three different compositions are shown: $4 \%$ singles (filled circle), 18\% singles (multi symbol), and 58\% singles (open box). Inset: percentage of all single balls that lie in each slice, on average

below the surface is larger than average, the number close to the surface will perforce be smaller than usual. The crossover from negative correlation occurs near the division between the grains that move during avalanches and those that do not.

We propose that the individual spheres rest in local formations of reduced stability, which begin to shift at an angle where the rest of the pile would still be immobile. The moving portion can then jar neighboring grains and initiate an avalanche. Similar formations may surround spheres deeper in the pile, but they are less free to begin moving as the mass above them increases. The large correlation between angle of stability and ball location for the $18 \%$ pile, which has the most even mixture of shapes in the relevant region of a few $\mathrm{cm}$ from the surface, supports this hypothesis. A configuration with more single balls near the surface is more likely to have at least one relatively unstable and unconstrained local arrangement, which would lead to an avalanche at a low angle. The $58 \%$ pile always has many single balls very close to the surface, leading to enough of the less stable formations near individual spheres that the existence of such regions is no longer a dominant factor in the angle of stability. At the other extreme, the $4 \%$ pile so seldom has single balls near the pile's surface that any unstable formations around single balls have little effect on the overall statistics.

A related explanation using surface traps has been used to describe some qualitatively similar results on monomer-dimer piles in three dimensions [34]. Like our work, these experiments find a slight nonlinearity of stability angle as a function of dimer weight fraction. A description in terms of surface traps, and the ability of particles to escape from such traps, shows reasonable agreement with the experiments [34].

\section{Conclusions}

We have measured stability for various mixtures of smooth small grains and less regular large grains. We find that radial segregation in our system is governed primarily by size difference rather than shape, with the smaller grains moving to the center of the container. We also find nonlinear behavior of the angle of stability, characterized by a sharp decrease when a few spheres are introduced into a homogeneous system of hexagons. We further show the importance of individual configurations. The spheres have much less influence on stability when confined to the outer edge of the container, and have the largest effect when they are positioned near the free surface of the pile. For mixtures of these two grain shapes, the locations of the spheres within the pile bears strongly on the overall stability. The enhanced influence of the spheres when they are near the free surface suggests that this portion of the pile is the most important in determining stability.

We plan to extend this work in several ways. By using faster rotation speeds we can observe dynamical angles of repose. It will be particularly interesting to see whether the hexagonal shapes still segregate to the outside of the container at high speeds. We will also examine in more detail how the segregation develops and how avalanches begin, and look at mixtures of other grain shapes. Finally, we will consider how to describe surface traps for hexagonal and other grain shapes.

Acknowledgments We thank D. de Guzman and T. Lin for their help with the image analysis.

Open Access This article is distributed under the terms of the Creative Commons Attribution Noncommercial License which permits any noncommercial use, distribution, and reproduction in any medium, provided the original author(s) and source are credited.

\section{References}

1. Duran, J.: Sands, Powders and Grains: An Introduction to the Physics of Granular Materials. Springer, New York (2000)

2. Jaeger, H.M., Nagel, S.R., Behringer, R.P.: Granular solids, liquids, and gases. Rev. Mod. Phys. 68, 1259-1273 (1996)

3. Daniels, K.E., Behringer, R.P.: Hysteresis and competition between disorder and crystallization in sheared and vibrated granular flow. Phys. Rev. Lett. 94, 168001-1-168001-4 (2005)

4. Deboeuf, S., Dauchot, O., Staron, L., Mangeney, A., Vilotte, J.-P.: Memory of the unjamming transition during cyclic tiltings of a granular pile. Phys. Rev. E 72, 051305-1-051305-11 (2005) 
5. Liu, C.-H.: Spatial patterns of sound propagation in sand. Phys. Rev. B 50, 782-794 (1994)

6. Ottino, J.M., Khakhar, D.V.: Mixing and segregation of granular materials. Annu. Rev. Fluid Mech. 32, 55-91 (2000)

7. Krishna, R., Ellenberger, J., Vandu, C.O.: Vibration-induced granular segregation in a pseudo-2D column: the (reverse) Brazil nut effect. Powder Technol. 164, 168-173 (2006)

8. Huerta, D.A., Ruiz-Suarez, J.C.: Vibration-induced granular segregation: a phenomenon driven by three mechanisms. Phys. Rev. Lett. 92, 114301-1-114301-4 (2004)

9. Carrigy, M.A.: Experiments on the angles of repose of granular materials. Sedimentology 14, 147-158 (1970)

10. Hill, K.M., Kakalios, J.: Reversible axial segregation of binary mixtures of granular materials. Phys. Rev. E 49, R3610-R3613 (1994)

11. Peratt, B.A., Yorke, J.A.: Continuous avalanche mixing of granular solids in a rotating drum. Europhys. Lett. 35, 31-35 (1996)

12. Hill, K.M., Caprihan, A., Kakalios, J.: Bulk segregation in rotated granular material measured by magnetic resonance imaging. Phys. Rev. Lett. 78, 50-53 (1997)

13. Choo, K., Molteno, T.C.A., Morris, S.W.: Traveling granular segregation patterns in a long drum mixer. Phys. Rev. Lett. 79, 2975-2978 (1997)

14. Lai, P.-Y., Jia, L.-C., Chan, C.K.: Friction induced segregation of a granular binary mixture in a rotating drum. Phys. Rev. Lett. 79, 4994-4997 (1997)

15. Ristow, G.H., Nakagawa, M.: Shape dynamics of interfacial front in rotating cylinders. Phys. Rev. E 59, 2044-2048 (1999)

16. Prigozhin, L., Kalman, H.: Radial mixing and segregation of a binary mixture in a rotating drum: Model and experiment. Phys. Rev. E 57, 2073 (1998)

17. Yanagita, T.: Three-dimensional cellular automaton model of segregation of granular materials in a rotating cylinder. Phys. Rev. Lett. 82, 3488-3491 (1999)

18. Puri, S., Hayakawa, H.: Segregation of granular mixtures in a rotating drum. Physica A 290, 218-242 (2001)

19. Newey, M., Losert, W.: Band-in-Band segregation of multidisperse granular mixtures. Europhys. Lett. 66, 205-211 (2004)
20. Kuo, H.P., Hsu, R.C., Hsiao, Y.C.: Investigation of axial segregation in a rotating drum. Powder Technol. 153, 196-203 (2005)

21. Liu, X.Y., Specht, E., Mellmann, J.: Experimental study of the lower and upper angles of repose of granular material. Powder Technol. 154, 125-131 (2005)

22. Taberlet, N., Newey, M., Richard, P., Losert, W.: On axial segregation in a tumbler: an experimental and numerical study. J. Stat. Mech. (2006). doi:10.1088/1742-5468/2006/07/P07013

23. Makse, H.A., Havlin, S., King, P.R., Stanley, H.E.: Spontaneous stratification in granular mixtures. Nature 386, 379-381 (1997)

24. Makse, H.A., Ball, R.C., Stanley, H.E., Warr, S.: Dynamics of granular stratification. Phys. Rev. E 58, 3357-3367 (1998)

25. Koeppe, J.P., Enz, M., Kakalios, J.: Phase diagram for avalanche stratification of granular media. Phys. Rev. E 58, R4104-R4107 (1998)

26. Rankenburg, I.C., Zieve, R.J.: The influence of shape on ordering of granular systems in two dimensions. Phys. Rev. E 63, 06130-1061303-9 (2001)

27. Albert, R., Albert, I., Hornbaker, D., Schiffer, P., Barabási, A.-L.: Maximum angle of stability in wet and dry spherical granular media. Phys. Rev. E 56, R6271-R6274 (1997)

28. Olson, J., Priester, M., Luo, J., Chopra, S., Zieve, R.J.: Packing fractions and maximum angles of stability in granular materials. Phys. Rev. E 72, 031302-1-031302-5 (2005)

29. Original images available at arXiv:0706.0774v1

30. Middleton, G.V., Hampton, M.A.: Marine sediment transport and environmental management, pp. 197-239. Wiley, New York (1976)

31. Brown, R.L., Richard, J.C.: Principles of Powder Mechanics, vol. 31. Pergamon, New York (1960)

32. Rajchenbach, J.: Flow in powders: From discrete avalanches to continuous regime. Phys. Rev. Lett. 65, 2221-2224 (1990)

33. Cantelaube, F., Limon-Duparcmeur, Y., Bideau, D., Ristow, G.H.: Geometrical analysis of avalanches in a 2D drum. J. Phys. (France) I 51, 581-596 (1995)

34. Ertag, D., Halsey, T.C., Levine, A.J., Mason, T.G.: Stability of monomer-dimer piles. Phys. Rev. E 66, 051307-1-051307-10 (2002) 